\title{
A Novel Approach in Improving Data Mining using Comparative Method of FPM
}

\author{
Darshana Bendwal \\ M.Tech Research Scholar \\ CSE, LNCT \\ Indore, MP, India
}

\author{
Santosh Varshney \\ Asst. professor \\ CSE, LNCT \\ Indore, MP, India
}

\begin{abstract}
Data mining is the procedure of distinguishing helpful example from expansive measure of information. Web mining is the procedure of discovering web design from web information. Web connection mining uses information from $\log$ record. Web connection mining discovering helpful things from $\log$ record. Log record contains all the client activities. In existing framework all the information are mine by apriori calculation. It is utilized for discovering succession from log record however it doesn't ordered log information as per our need. Bolster vector machine is utilized to group all the information of web log document that examine information and recognize designs. Bolster vector machine order all the information into two classes. It separates two classes by hyper plane. In the wake of applying SVM, We find all the information into one example. This example is helpful for business investigator to take a valuable choice. At long last we get arrange information from web route information. Information Mining is recovery of Knowledge from a lot of information. A Frequent example is an example that shows up in information set every now and again. It might be an item set, subsequence or substructures. An arrangement of things that show up as often as possible together in a value-based database is called Frequent Itemset. Successive Itemset Mining is the key venture in affiliation standard mining and in discovering relationships. FPM additionally assumes an imperative part in recognizing fascinating connections among information. The identification of intriguing connection connections among extensive business exchange tuples can help in choice making methodology and client shopping conduct investigation (i.e., market-wicker container examination). FPM helps the business individuals to create showcasing procedures for picking up benefits. There are numerous calculations that have been proposed for finding continuous itemset mining in a value-based dataset. They are Apriori, FP-Growth, Vertical Partitioning, RELIM and so on. In this paper, I look at the adequacy of these calculations and proposed another calculation in a propelled methodology. The new thought about this calculation is gotten from existing calculations. The viability of this new calculation can be accomplished with less number of outputs and better halfway steps.[1]
\end{abstract}

\section{Keywords}

Apriority, SVM classifier, Web Link Mining, Log File, Pattern recognition

\section{INTRODUCTION}

Development of information from boundless measures of information is called information mining or learning extraction. One of the information mining functionalities is affiliation standard mining. The information is as value-based or social Macintosh, use the font named Times. Right margins should be justified, not ragged. model. Affiliation standard mining is useful in discovering the regular thing set in the value-based database.

Network Information mining is the process utilized for give the valuable learning and separating the fascinating example from the immense measure of information set[2] .

Successive examples are thing sets, subsequence, or substructures that show up in an information set with recurrence no not as much as a client indicated edge. Successive example mining is an initial phase in affiliation guideline mining.[Comparative Analysis of Various Approaches Used in Frequent Pattern Mining] One of the most regular clients of continuous mining is to investigation of substantial measure of information. It is likewise called as web information. There are taking after sort of web information in web mining [3].

\subsection{Web Server Data:}

Client $\operatorname{logs}$ are accumulated by the web server and consistently fuse IP area, page reference and access time.

\subsection{Application Server Data:}

Business procurement servers, for instance, Web logic, Story server, have enormous attributes to enable E-business orders to be in view of top of them with little effort. A key trademark

Is the ability to track diverse sorts of business events and log them in demand server logs.

\subsection{Application Level Data:}

New sorts of events could be portrayed in a procurement, and logging may be turned on for them creating histories of these events. It must be noted, regardless, that various end procurements oblige a combo of one or a more noteworthy measure of the frameworks associated in the over the arrangement 


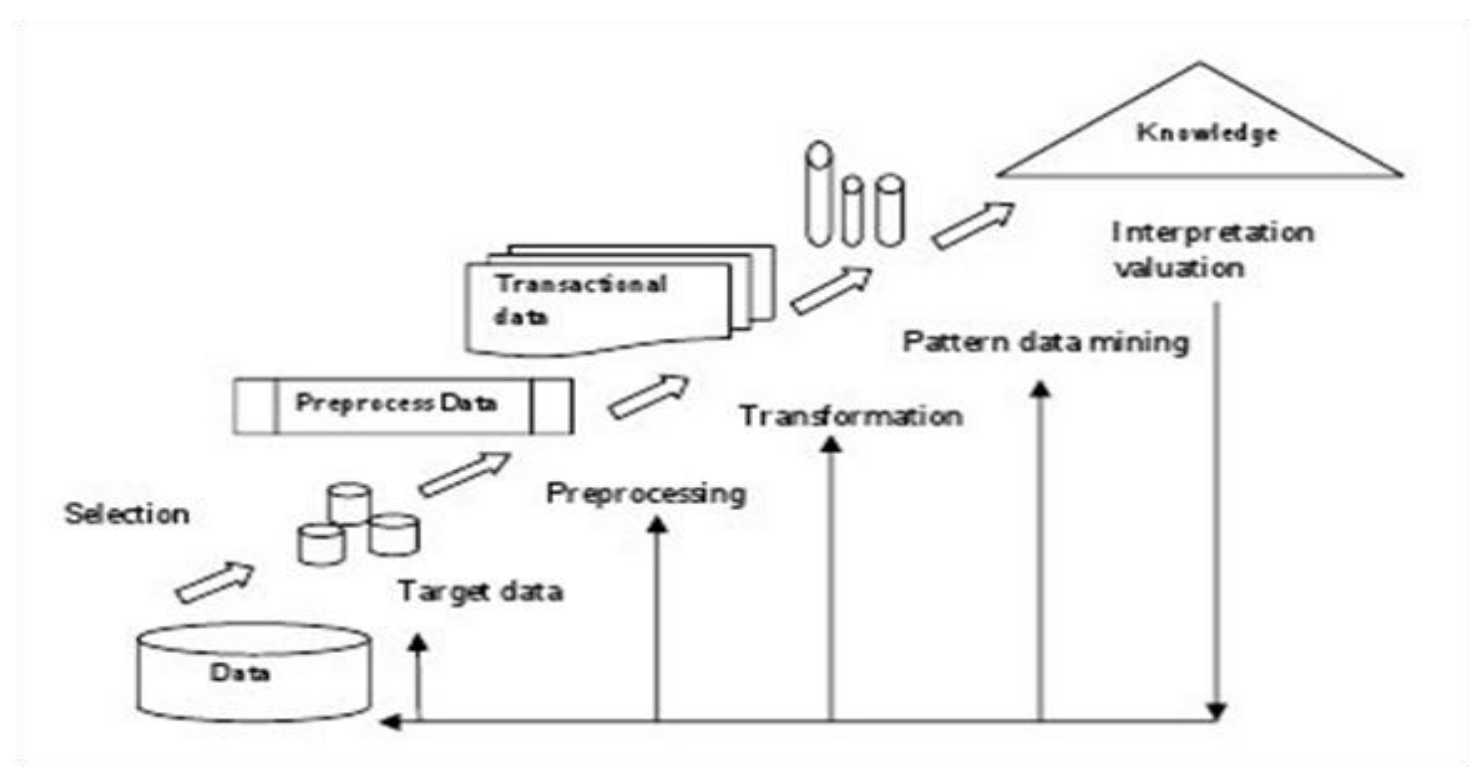

Fig 1: The move from crude information to important learning

\section{LITERATURE SURVEY}

The current examination works is separated into three principle classes an) Association Rule Mining (ARM), b) Clustering, c) Classification.

In Association Rule Mining, Ming-Syan et al., [4] proposed another information mining calculation that includes mining way traversal examples in an appropriated data giving environment where archives or items are connected together to encourage intuitive access. Jianhan Zhu et al., connected the Markov binds to model client navigational conduct. They proposed a strategy for developing a Markov model of a site in light of past guest conduct. At that point the Markov model is utilized to make join expectations that aid new clients to explore the Web webpage. client's examples. Mehrdad Jalali et al., [displayed a methodology which is in view of the chart parceling for demonstrating client route designs. Keeping in mind the end goal to mining client route designs, they make an undirected chart in view of integration between every pair of the website pages furthermore proposed novel equation for allotting weights to edges of the diagram.

In Classification, Mahdi Khosravi et al., [6] proposed a novel methodology for element mining of clients' advantage route examples, utilizing guileless Bayesian technique.

\section{VARIOUS APPROACH OF FPM}

\subsection{Apriori Based Algorithms:}

It is a first calculation for continuous example mining. By utilizing thing set's descending conclusion property apriori calculation diminish the extent of pursuit space. On the off chance that a thing arrangement of length $\mathrm{k}$ is not visit, none of its superset examples can be visit. Applicant incessant thing sets, $\mathrm{Ck}$ where $\mathrm{k}$ is the length of the thing set, are produced before every information examine. The backings of hopeful successive thing sets are numbered. Competitor $\mathrm{k}$ thing sets, $\mathrm{Ck}$ are produced with regular $(\mathrm{k}-1)$ thing sets. Execution of apriori is increment by lessening the hopeful set. Toward the end of every sweep, exchanges that are conceivably helpful.

\begin{tabular}{|l|l|l|l|l|}
\hline Techniques & $\begin{array}{l}\text { Apriori } \\
\text { Based }\end{array}$ & $\begin{array}{l}\text { Partition } \\
\text { Based }\end{array}$ & FP Tree & $\begin{array}{l}\text { SQL } \\
\text { Based }\end{array}$ \\
\hline $\begin{array}{l}\text { Number of } \\
\text { Database } \\
\text { scan (Best } \\
\text { Case) }\end{array}$ & 2 & 1 & 2 & 1 \\
\hline $\begin{array}{l}\text { Number of } \\
\text { Database } \\
\text { scan (Worst } \\
\text { Case) }\end{array}$ & A+1 & $(2 \mathrm{~B}-1) / \mathrm{B}$ & 2 & 1 \\
\hline $\begin{array}{l}\text { Candidate } \\
\text { Generation } \\
\text { Needed or } \\
\text { Not? }\end{array}$ & Yes & Yes & No & No \\
\hline $\begin{array}{l}\text { Incremental } \\
\text { Mining } \\
\text { Possible }\end{array}$ & No & No & No & No \\
\hline $\begin{array}{l}\text { Sensitive to } \\
\text { Change in } \\
\text { User } \\
\text { Parameter }\end{array}$ & Yes & Yes & Yes & Yes \\
\hline
\end{tabular}

\subsection{Part based Algorithms:}

Part-Based calculation is to conquer the issue of high number of database outputs. In this Algorithm entire information set is sweep in only two sweeps. In this calculation dataset is partitioning into numerous number of little dataset. In First information check, calculation make nearby incessant thing set in every allotment. Since entire parcel can be put away into primary memory mining methodology managed with no plate I/O operation. The neighborhood visit thing sets are included into the worldwide competitor's successive thing set. In second step, false applicants are disposed of from the worldwide hopefuls visit thing set. By applying past learning 
amid the competitor thing set extricating procedure. Is will help in discovering the false worldwide applicant incessant thing set. False thing set then expelled from rundown. In most pessimistic scenario, number of output for allotment base calculation is $(2 b-1) / b$ where $b$ is number of part [8].

\subsection{Design development Algorithm:}

There are two primary disadvantage of apriori calculation one is create applicant continuous thing set and other is expense identified with I/O operation. Second issue can be unravel yet for first issue Han et al. proposed an information structure called incessant example tree or FP Tree. FP-development mines visit thing sets from FP-Tree without producing applicant incessant thing sets. It is an augmentation of prefix tree structure. Every hub of tree contain thing mark and its recurrence. Bolster worth characterized the foundation of thing in tree structure root to leave. All filtering procedure is finished in two stages. In first step, this compute the bolster esteem and in second sweep, this check is utilized to sort the thing in dropping request. On the off chance that two exchanges impart a typical prefix, the imparted segment is combined and the frequencies of the hubs are increased as needs be. Hubs with the same name are associated with a thing connection. The thing connection is utilized to encourage successive example mining. In this method we guarantee that all example set are produce legitimately. There are two primary downside of apriori calculation one is create competitor visit thing set and other is expense identified with I/O operation. Second issue can be comprehend however for first issue Han et al. proposed an information structure called continuous example tree or FP Tree. FP-development mines visit thing sets from FP-Tree without creating applicant continuous thing sets. It is an augmentation of prefix tree structure. Every hub of tree contains thing mark and its recurrence. Bolster quality characterized the foundation of thing in tree structure root to leave. All examining methodology is finished in two stages. In first step, this compute the bolster esteem and in second sweep, this number is utilized to sort the thing diving request. [9]

Trans ID List of Item IDs

T1 11,12,14,15,16,20,22

T2 11, 16, 17, 25

T3 13, 15, 18, 19

T4 11, 13, 14, 15, 17, 19

T5 $11,12,13,15,17,21$

T6 15, 18, 23

T7 11, 12, 13, 15, 1620

T8 11, 13, 14, 21

T9 $11,13,15,17,23$

$\mathrm{T} 1011,13,15,17,24$

and $\operatorname{Sup} \min =4$.

\subsubsection{Solution using apriori}

\section{C1}

$\begin{array}{lc}\text { Item set } & \text { Support count } \\ 11 & 8 \\ 12 & 3 \\ 13 & 7 \\ 14 & 3 \\ 15 & 8 \\ 16 & 3\end{array}$

$\begin{array}{ll}17 & 5 \\ 18 & 2 \\ 19 & 2 \\ 20 & 2 \\ 21 & 2 \\ 22 & 1 \\ 23 & 2 \\ 24 & 2 \\ 25 & 1 \\ \end{array}$

\section{$\mathrm{C} 2=\mathrm{L} 1 \times \mathrm{L} 1$}

L2

Item set

Support count

11,13

11,15

11,17

6

11,17

6

13,15

5

13,17

6

15,17

4

$\mathrm{C} 3=\mathrm{L} 2 \times \mathrm{L} 2$

Item set

$11,13,15$

$11,13,17$

$11,15,17$

$13,15,17$

C4

Item set

$11,13,15,17$

Support Count

4

\subsubsection{Fp growth}

The first scan of the database is same as apriori. An FP-Tree is constructed with root as null. The set of frequent items sorted in the descending support count and denote it as $\mathrm{L}$.

$\mathrm{L}=\{\langle 11: 8\rangle,\langle 15: 8\rangle,\langle 13: 7\rangle,\langle 17: 5\rangle\}$

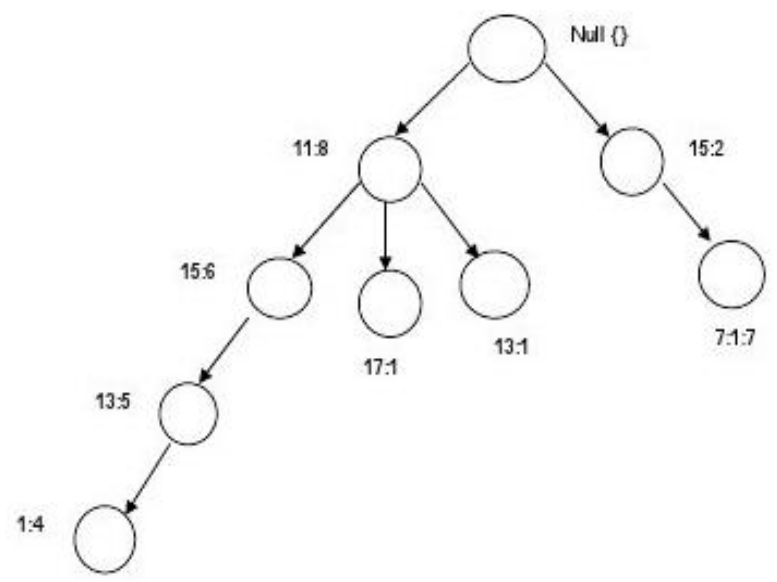

Fig 2: FP-Tree Construction

FP-Growth technique changes the issue of finding long successive examples to looking for shorter examples recursively and after that connecting the postfix. There is no hopeful era, yet the pointers need to store in memory oblige vast space. This system extensively lessens the inquiry cost. Alternate strategies, for example, Vertical information 
configuration and RELIM have their own particular inadequacies.

\section{- Vertical Layout}

It is quicker than apriori. Change the evenly arranged information into vertical configuration by checking the information set once. The bolster tally of an item set is the length of TID_set of the item set. TID_set takes considerable memory \& time for the set convergence and likewise require more memory space contrasted and flat design.

\section{- RELIM (Recursive Elimination)}

It calculation works better than all the prior calculations and is fundamentally enlivened by FP-Growth calculation. There is no competitor era anyhow, it obliges preprocessing on database in the first and foremost stage which makes more computational expense. The primary quality of this calculation is not speed, but rather the straightforwardness of its structure.[10]

\section{SUGGESTED APPROACH}

1. Examine the Database "D"

2. Compute Frequent 1-Itemset

3. Sort the 1-itemset by rising request of backing (if the things are of same backing organize them either in climbing request or in sequential order way)

4. Develop Frequent-2 table by right neighbor system and output the database

5. Develop the Tree with double values (strings) in every leaf.

6. $\mathrm{P}=3$

While \{height of the tree expanded

Produce P-layer Tree ( )

$\mathrm{P}=\mathrm{P}+1$

\}

7. List the successive things from the tree.

\section{CONCLUSION}

In the new approach, there is no competitor situated era furthermore in Frequent- 2 table, the summation of each segment information gives the bolster tally. There are no complex tree traversal techniques. Bitwise AND operation discovers the bolster include of new hub a quick way, at the point when contrasted and Apriori, FP-Growth, Vertical Parceling and RELIM. The two essential FPM procedures specifically Apriori and FP-Growth are successfully coordinated. In this paper, different methods for regular example mining are clarified. The strategies are chosen based upon its application. Yield issues us incessant example specifically session. There are such a variety of looks into going on example mining yet some more research is needed, in light of the fact that in today's reality conduct of client is all that much essential for site. Discovering incessant thing set is extremely valuable in different errands, for example, select time of site upkeep, discover most went by page of site, discovering spot for post promotion and so on.[11]

\section{ACKNOWLEDGEMENT}

The work is evaluated and drafted with the help of some of authorities of the Lakshmi Narain College of Technology Institute which leads me to the great outcomes. Without them it would not be possible for me to overcome the problems and issues faced. Thus, the authors thank the anonymous reviewers for their valuable comments, which strengthened the paper. They also like to give thanks to Assistant professor Santosh Varshney who had guided me throughout this research and being held always for discussion regarding the approach adapted for this paper.

\section{REFERENCES}

[1] Paweł Weichbroth, Mieczysław Owoc," Web User Navigation Patterns Discovery from WWW Server Log Files", 978-83-60810-48-4, 2012 IEEE.

[2] Mr. Akshay A. Adsod, Prof. Nitin R. Chopde, "A Review on: Web Mining Techniques", ISSN: 2231-5381 IJETT - Volume 10 Number 3 - Apr 2014.

[3] Ming-Syan Chen, Jong Soo Park, Philip S. Yu, "Efficient Data Mining for Path Traversal patterns", ieee transactions on knowledge and data engineering, vol. 10, no. 2, march/april 1998.

[4] C.Borgelt. 2003. Efficient Implementations of Apriori and Eclat. In Proc. 1st IEEE ICDM Workshop on Frequent Item Set Mining Implementations, CEUR Workshop Proceedings 90, Aachen, Germany.

[5] Agarwal, R. C., Agarwal, C. C. and Prasad, V. V. V. (2001) A tree projection algorithm for generation of frequent item sets. Journal of Parallel and Distributed Computing, 61(3), Pp. 350-371.

[6] Deepak Garg et. al. "Comparative Analysis of Various Approaches Used in Frequent Pattern Mining" (IJACSA) International Journal of Advanced Computer Science and Applications, Special Issue on Artificial Intelligence.

[7] Han.J., Pei.J., Yin. Y.2000. Mining frequent patterns without candidate generation. In Proc. ACM-SIGMOD Int'l Conf. Management of Data (SIGMOD).

[8] J. Pei, J. Han; R. Mao. 2000. Closet: An efficient algorithm for mining frequent closed itemsets. In ACM SIGMOD Workshop on Research Issues in Data Mining and Knowledge Discovery.

[9] Goswami D. N et. al. "An Algorithm for Frequent PatternMining Based On Apriori " (IJCSE) International Journal on Computer Science and Engineering Vol. 02, No. 04, 2010, Pp. 942-947.

[10] D. Burdick, M. Calimlim, J. Flannick, J. Gehrke, T. Yiu.Nov 2005. Mafia: a maximal frequent itemset algorithm. IEEE Transactions on Knowledge and Data Engineering, vol., no., pp. 1490-1504, 17(11).

[11] Agrawal.R., Srikant.R. September 1994. Fast algorithmsfor mining association rules. In Proc. Int‘l Conf. Very Large Data Bases (VLDB), pp.487-499. 